\title{
Paradoxical response to disseminated non-tuberculosis mycobacteriosis treatment in a patient receiving tumor necrosis factor-a inhibitor: a case report
}

\author{
Takahiro Takazono ${ }^{1}$, Shigeki Nakamura ${ }^{1 *}$, Yoshifumi Imamura ${ }^{1}$, Taiga Miyazaki', Koichi Izumikawa' \\ Hiroshi Kakeya ${ }^{1,3}$, Katsunori Yanagihara ${ }^{2}$ and Shigeru Kohno ${ }^{1}$
}

\begin{abstract}
Background: Biological agents such as tumor necrosis factor-a inhibitors are known to cause mycobacterium infections. Here, we report a disseminated non-tuberculosis case caused by TNF-a inhibitor therapy and a probable paradoxical response to antimycobacterial therapy.

Case presentation: A 68-year-old man with relapsing polychondritis was refractory to glucocorticoid therapy; adalimumab was therefore administered in combination with oral glucocorticoids. Treatment with $40 \mathrm{mg}$ of adalimumab led to rapid improvement of his clinical manifestations. The administration of tacrolimus (1 mg) was started as the dosage of oral glucocorticoids was tapered. However, the patient developed an intermittent high fever and productive cough 15 months after starting adalimumab treatment. A chest computed tomography scan revealed new granular shadows and multiple nodules in both lung fields with mediastinal lymphadenopathy, and Mycobacterium intracellulare was isolated from 2 sputum samples; based on these findings, the patient was diagnosed with non-tuberculosis mycobacteriosis. Tacrolimus treatment was discontinued and oral clarithromycin (800 mg/day), rifampicin (450 mg/day), and ethambutol $(750 \mathrm{mg} /$ day) treatment was initiated. However, his condition continued to deteriorate despite 4 months of treatment; moreover, paravertebral and subcutaneous abscesses developed and increased the size of the mediastinal lymphadenopathy. Biopsy of the mediastinal lymphadenopathy and a subcutaneous abscess of the right posterior thigh indicated the presence of Mycobacterium avium complex (MAC), and the diagnosis of disseminated non-tuberculosis mycobacteriosis was confirmed. Despite 9 months of antimycobacterial therapy, the mediastinal lymphadenopathy and paravertebral and subcutaneous abscesses had enlarged and additional subcutaneous abscesses had developed, although microscopic examinations and cultures of sputum and subcutaneous abscess samples yielded negative results. We considered this a paradoxical reaction similar to other reports in tuberculosis patients who had discontinued biological agent treatments, and increased the dose of oral glucocorticoids. The patient's symptoms gradually improved with this increased dose and his lymph nodes and abscesses began to decrease in size.

(Continued on next page)
\end{abstract}

\footnotetext{
* Correspondence: moju516@nagasaki-u.ac.jp

'Department of Molecular Microbiology and Immunology, Nagasaki University Graduate School of Biomedical Sciences, Nagasaki University School of Medicine, Nagasaki, Japan

Full list of author information is available at the end of the article
} 
(Continued from previous page)

Conclusions: Clinicians should consider the possibility of a paradoxical response when the clinical manifestations of non-tuberculosis mycobacteriosis worsen in spite of antimycobacterial therapy or after discontinuation of tumor necrosis factor-a inhibitors. However, additional evidence is needed to verify our findings and to determine the optimal management strategies for such cases.

Keywords: Non-tuberculosis mycobacteriosis, Mycobacterium intracellulare, Disseminated infection, Relapsing polychondritis, Paradoxical response, Tumor necrosis factor-a inhibitor, Immune reconstitution inflammation syndrome, Non-HIV

\section{Background}

Relapsing polychondritis (RP) is a rare systemic disorder characterized by inflammation of the bilateral auricular or tracheal cartilage. Standard treatments for RP include immunosuppressive agents such as corticosteroids, biological agents such as tumor necrosis factor- $\alpha$ (TNF- $\alpha$ ) inhibitors and anti-inflammatory agents commonly used to treat rheumatoid arthritis [1]. Patients receiving biological agents have an increased risk for opportunistic mycobacterial and fungal infections [2]. In particular, patients with non-tuberculosis mycobacteriosis (NTM) infections who receive treatments with biological agents are more likely to develop extra-pulmonary or disseminated infections compared with immunocompetent patients [3]. Although biological agents are contraindicated for patients with known NTM, it is difficult to completely eliminate the possibility of infection before starting therapy.

Furthermore, there have been reports of paradoxical responses in patients who developed tuberculosis after discontinuation of anti-TNF- $\alpha$ agents $[4,5]$. Here, we report a case of disseminated NTM with RP caused by TNF- $\alpha$ inhibitor therapy and a probable paradoxical response to antimycobacterial therapy.

\section{Case presentation}

A 68-year-old man was referred to our hospital for a fever of unknown origin. His medical history was unremarkable except for hypertension. The presence of bilateral auricularis and nasal root inflammation, along with auricular perichondritis that was positive for anti-type II collagen antibodies, led to a diagnosis of RP. Because he was refractory to prednisolone (PSL) glucocorticoid therapy at a dose of $30 \mathrm{mg} /$ day, adalimumab was initiated in combination with oral glucocorticoids after a chest computed tomography (CT) scan indicated that he had no signs of tuberculosis or NTM. Interferon- $\gamma$ (IFN- $\gamma$ ) release assays (T-SPOT. TB, Oxford Immunotec Ltd., Massachusetts, US) and anti-Mycobacterium avium complex (MAC) antibody assays (Capilia MAC, TAUNS laboratories, Inc., Shizuoka, Japan) indicated also negative results. After starting adalimumab treatment $(40 \mathrm{mg})$, his clinical manifestations rapidly improved; therefore, adalimumab was administered 3 times approximately every 2 weeks. The clinical manifestations of RP resolved; moreover, while the PSL dose was gradually tapered to $10 \mathrm{mg} /$ day, treatment with tacrolimus ( $1 \mathrm{mg} /$ day) was introduced.

The patient subsequently exhibited an intermittent high fever and productive cough 16 months after the RP diagnosis. Laboratory tests showed a normal white blood cell count $\left(8,100 / \mathrm{mm}^{3}\right)$ and procalcitonin concentration (0.099 ng/mL), and increased C-reactive protein levels (13.81 mg/dL, normal range $<0.3 \mathrm{mg} / \mathrm{dL}$ ). The results of all other laboratory tests including liver enzymes, creatinine, and blood urea nitrogen were within normal ranges. A chest CT scan showed granular shadows and multiple nodules in both lung fields with mediastinal lymphadenopathy (Figure 1). Mycobacterium intracellulare was isolated from 2 sputum samples; based on these findings, the patient was diagnosed with a pulmonary infection with this NTM. The minimum inhibitory concentrations of the isolated strain for clarithromycin (CAM), rifampicin (RIF), and ethambutol (EMB) were $0.5,32.0$, and $8.0 \mu \mathrm{g} / \mathrm{mL}$, respectively. Tacrolimus treatment was discontinued. Treatments with CAM, RIF, and EMB at 800, 450, and $750 \mathrm{mg} /$ day, respectively were initiated. The size of the pulmonary nodules and mediastinal lymphadenopathy increased 1 month after the initiation of antimycobacterial therapy. The high fever and general fatigue worsened despite 4 months of treatment; paravertebral and subcutaneous abscesses also developed and the size of the mediastinal lymphadenopathy increased.

Because biopsy of the mediastinal lymphadenopathy and a subcutaneous abscess of the right posterior thigh indicated infection by $M$. intracellulare, the diagnosis of disseminated MAC infection was confirmed. After 5 months of antimycobacterial therapy with CAM, RIF, and EMB, we changed the medication from RIF to rifabutin at $300 \mathrm{mg} /$ day, increased the dose of CAM from 800 to $1,000 \mathrm{mg} /$ day, and added streptomycin at $0.75 \mathrm{~g}, 3$ times per week; however, the abscesses continued to enlarge. Tests for anti-human T-cell leukemia virus-1 antibodies, anti-human immunodeficiency virus (HIV) antibodies, and antibodies to IFN- $\gamma$ all yielded negative results. Although we slowly decreased the dose of PSL to $5 \mathrm{mg} /$ day, the mediastinal lymph nodes enlarged even further and abscesses were formed. The mediastinal lymphadenopathy 


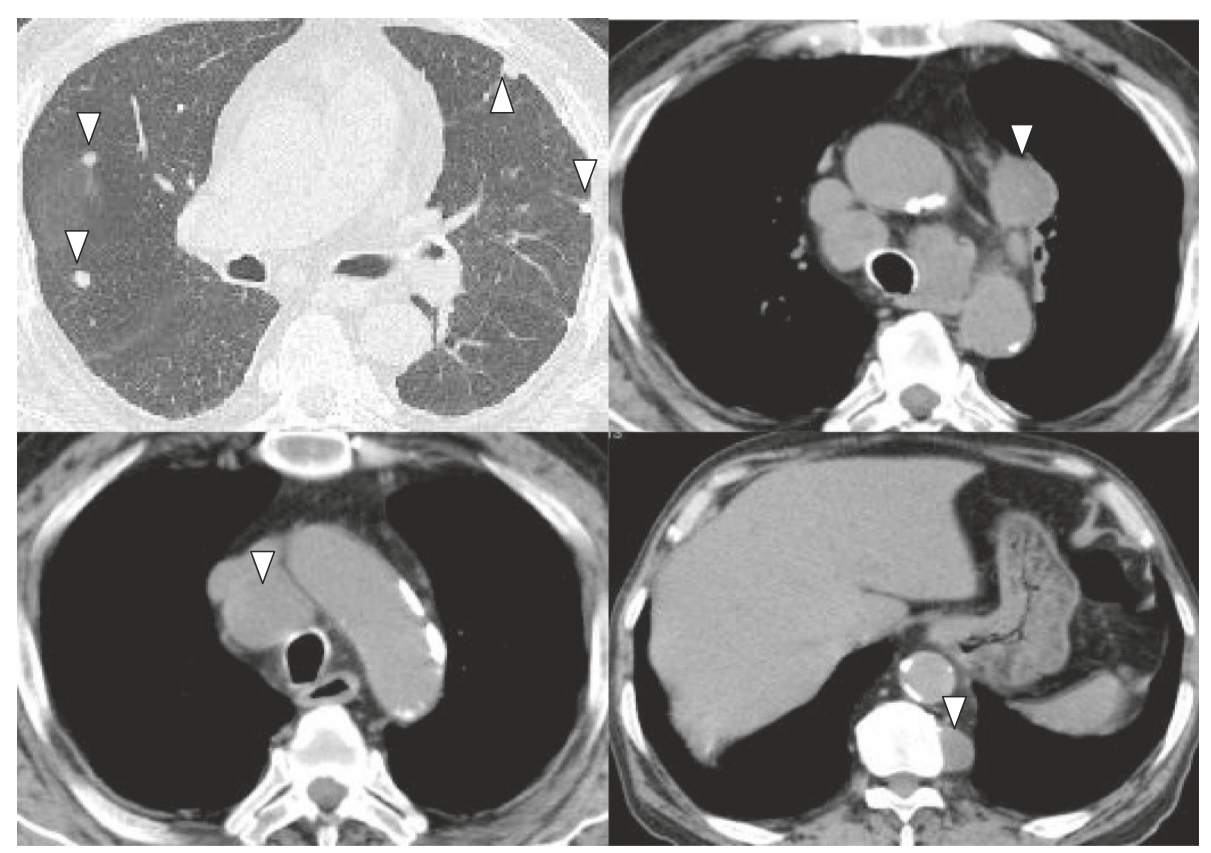

Figure 1 Computed tomography images of disseminated NTM development. The white triangles indicate pulmonary nodules, mediastinal lymph nodes, and paravertebral abscess.

and paravertebral and subcutaneous abscesses also enlarged more and other subcutaneous abscesses developed, although microscopic examination and culture results of sputum and subcutaneous abscess samples indicated negative results 9 months after induction of antimycobacterial therapy. We considered this a paradoxical reaction similar to that noted in previous reports in patients with tuberculosis who discontinued biological agent therapy; therefore, we increased the PSL dosage to $10 \mathrm{mg} /$ day. After this increase, his symptoms gradually improved and the lymph nodes and abscesses began to decrease in size. Due to the deterioration of RP symptoms, the PSL dosage was increased to $30 \mathrm{mg} /$ day and mizoribine was added as a treatment; however, the MAC infection lesions continued to decrease in size. The clinical course, culture results, treatment regimens, and CT scan images are shown serially in Figure 2.

\section{Discussion and Conclusions}

RP itself is not generally considered a risk factor for mycobacterial infections since there have been no reports of RP complicated with NTM. Reports in Thailand and Taiwan found that that most non-HIV patients with disseminated NTM infections exhibited positive results for IFN- $\gamma$ autoantibodies [6]; however, the patient in the present case exhibited negative results for these antibodies. Patients receiving biological agents are likely to develop extra-pulmonary or disseminated NTM [3], and several fetal cases have been reported [7-9]. Therefore, the disseminated NTM infection in this case was suspected to be related to the use of a TNF $\alpha$ inhibitor.

Mori, et al. reviewed 13 pulmonary NTM cases that had received biological therapy for $\mathrm{RA}$, including infliximab, etanercept, adalimumab, and tocilizumab [10]. The average time from the initiation of biological agent therapy to development of NTM was 10 months (range: 6 weeks to 8.5 years), similar to our case (15 months). Although no cases of disseminated NTM were reported, most cases responded appropriate anti-NTM treatment following discontinuation of biological agents, and none of the cases had a paradoxical reaction after initiation of anti-NTM treatment [10]. Furthermore, in one case, restarting tocilizumab therapy while continuing antiNTM therapy resulted in a favorable outcome $[10,11]$.

The paradoxical worsening of clinical symptoms and radiologic findings despite effective antimycobacterial therapy may be attributed to immune reconstitution inflammatory syndrome (IRIS). IRIS is defined as the worsening or recurrence of inflammatory symptoms during restoration of an effective immune response against pathogens of opportunistic infections [12,13]. The syndrome has primarily been reported in patients infected with HIV who experience immune reconstitution after initiation of antiretroviral therapy.

It has been suggested that anti-TNF- $\alpha$ agents should be discontinued when Mycobacterium tuberculosis infection occurs. However, IRIS has been reported in patients with tuberculosis after discontinuation of anti-TNF- $\alpha$ 


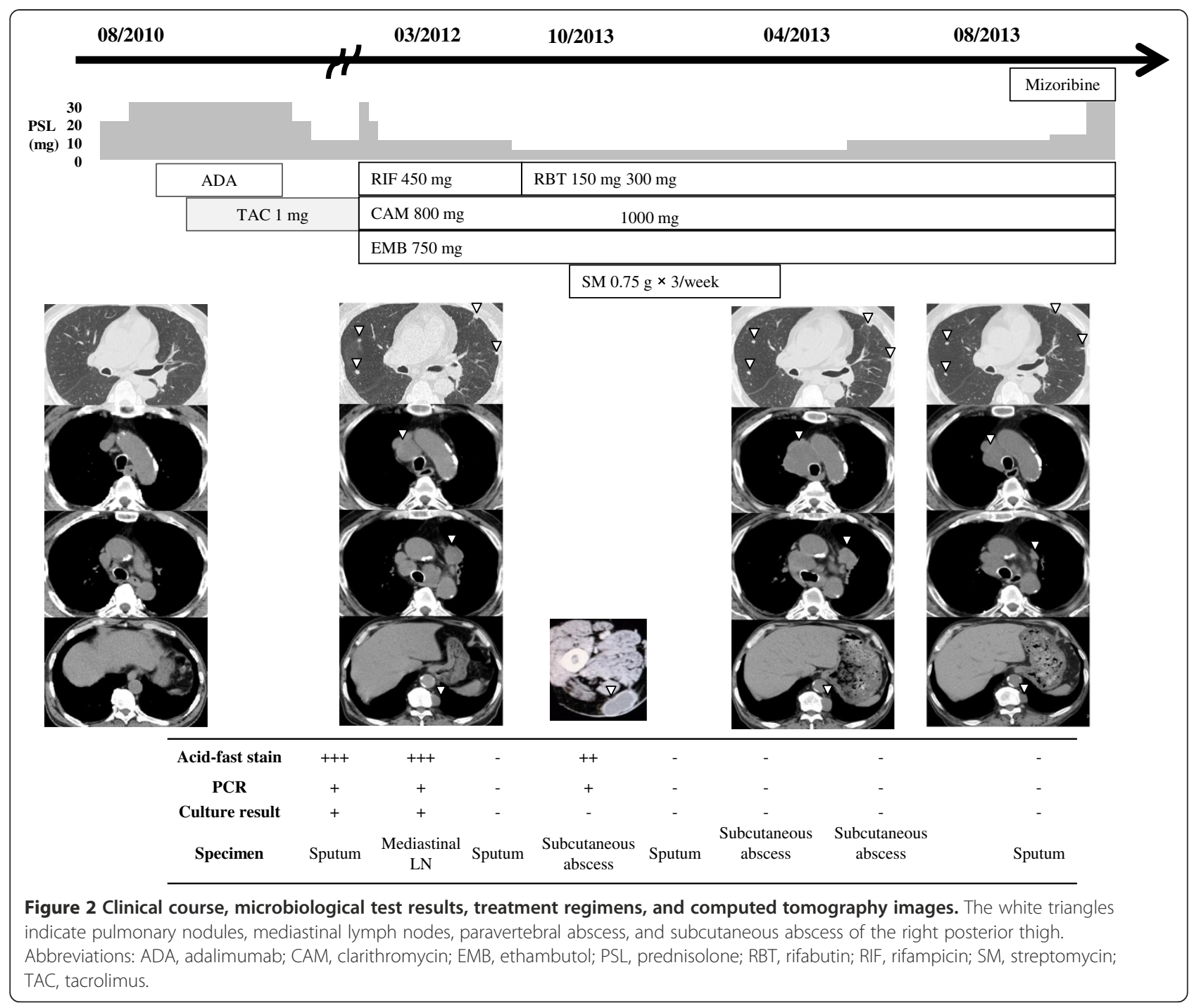

agents $[4,5]$. Furthermore, resumption of anti-TNF- $\alpha$ with antimycobacterial drug therapy has been reported to be effective in a tuberculosis case exhibiting a paradoxical response [14]. There are no guidelines or sufficient evidence to recommend a specific course of action in tuberculosis patients administered TNF- $\alpha$ inhibitors who developed a paradoxical response while receiving antimycobacterial treatment.

The reasons for this paradoxical reaction to anti-NTM treatment remain unclear. The combination of adalimumab, tacrolimus, and prednisolone therapies might have resulted in a high mycobacterial load that provoked an immune/inflammatory response upon cessation of adalimumab and tacrolimus treatments.

To our knowledge, this is the first reported non-HIV case of NTM to develop a paradoxical response. Although we considered the presence of a paradoxical response in the present case, the resumption of biological agent treatment was risky because antimycobacterial therapy against NTM is not always effective. Because corticosteroids are an effective treatment for IRIS in patients with HIV, we chose to increase the dose of PSL. Fortunately, our patient responded to this increase, although evidence regarding the optimal dose and duration of steroid therapy remains unclear.

If clinical manifestations of infection worsen despite the administration of antimycobacterial therapy after discontinuation of anti-TNF- $\alpha$ agents, the possibility of a paradoxical response should be considered. Additional evidence is needed to verify our findings and to determine optimal management of such cases.

\section{Consent}

Written informed consent was obtained from the patient for publication of this Case Report and any accompanying images. A copy of the written consent is available for review by the Series Editor of this journal. 


\section{Competing interests}

The authors declare that they have no competing interests.

\section{Authors' contributions}

$\Pi$, SN, YI, TM, and HK were responsible for clinical testing and treatments. KI and SK supervised the work and helped to draft the manuscript. KY participated in the microbiological work-ups. All authors have read the manuscript and accepted the final version.

\section{Author details}

'Department of Molecular Microbiology and Immunology, Nagasaki University Graduate School of Biomedical Sciences, Nagasaki University School of Medicine, Nagasaki, Japan. ${ }^{2}$ Department of Laboratory Medicine, Nagasaki University Hospital, Nagasaki, Japan. ${ }^{3}$ Department of Infection Control Science, Graduate School of Medicine, Osaka University, Osaka City, Japan.

Received: 24 December 2013 Accepted: 24 February 2014

Published: 28 February 2014

\section{References}

1. Sharma A, Gnanapandithan K, Sharma K, Sharma S: Relapsing polychondritis: a review. Clin Rheumatol 2013, 32(11):1575-1583.

2. Listing J, Strangfeld A, Kary S, Rau R, Hinueber U, Scholz SM, Ihle EG, Antoni C, Herzer P, Kekow J, Schneider M, Zink A: Infections in patients with rheumatoid arthritis treated with biologic agents. Arthritis Rheum 2005, 52(11):3403-3412.

3. Winthrop KL, Chang E, Yamashita S, lademarco MF, LoBue PA: Nontuberculous mycobacteria infections and anti-tumor necrosis factor-alpha therapy. Emerg Infect Dis 2009, 15(10):1556-1561.

4. Moureau C, Pothen L, Wilmes D, Yombi JC, Coche E, Hainaut P: Paradoxical response to tuberculosis treatment in a patient receiving tumor necrosis factor-alpha antagonist. Am J Med 2012, 125(6):e9-e10.

5. Garcia Vidal C, Rodriguez Fernandez S, Martinez Lacasa J, Salavert M, Vidal R, Rodriguez Carballeira M, Garau J: Paradoxical response to antituberculous therapy in infliximab-treated patients with disseminated tuberculosis. Clin Infect Dis 2005, 40(5):756-759.

6. Browne SK, Burbelo PD, Chetchotisakd P, Suputtamongkol Y, Kiertiburanakul S, Shaw PA, Kirk JL, Jutivorakool K, Zaman R, Ding L, Hsu AP, Patel SY, Olivier KN, Lulitanond V, Mootsikapun P, Anunnatsiri S, Angkasekwinai N, Sathapatayavongs B, Hsueh PR, Shieh CC, Brown MR, Thongnoppakhun W, Claypool R, Sampalo EP, Thepthai C, Waywa D, Dacombe C, Reizes Y, Zelazny AM, Saleeb P, Rosen LB, Mo A, ladarola M, Holland SM: Adult-onset immunodeficiency in Thailand and Taiwan. N Engl J Med 2012, 367(8):725-734.

7. Besada E: Rapid growing mycobacteria and TNF-alpha blockers: case report of a fatal lung infection with Mycobacterium abscessus in a patient treated with infliximab, and literature review. Clin Exp Rheumatol 2011, 29(4):705-707.

8. Marie I, Heliot P, Roussel F, Herve F, Muir JF, Levesque H: Fatal Mycobacterium peregrinum pneumonia in refractory polymyositis treated with infliximab. Rheumatology (Oxford) 2005, 44(9):1201-1202.

9. Maimon N, Brunton J, Chan AK, Marras TK: Fatal pulmonary Mycobacterium xenopi in a patient with rheumatoid arthritis receiving etanercept. Thorax 2007, 62(8):739-740.

10. Mori S, Tokuda H, Sakai F, Johkoh T, Mimori A, Nishimoto N, Tasaka S, Hatta K, Matsushima H, Kaise S, Kaneko A, Makino S, Minota S, Yamada T, Akagawa S, Kusashima A, NTM-BIORA (NTM infection in Biologic-treated RA patients) Study Investigators: Radiological features and therapeutic responses of pulmonary nontuberculous mycobacterial disease in rheumatoid arthritis patients receiving biological agents: a retrospective multicenter study in Japan. Mod Rheumatol 2012, 22(5):727-737.

11. Nakahara H, Kamide $Y$, Hamano $Y$, Hosokawa T, Nishide M, Lin Y, Kawamoto K, Fusama M, Higa S, Kuroiwa T, Igarashi T, Kuritani T, Maeda K: A case report of a patient with rheumatoid arthritis complicated with Mycobacterium avium during tocilizumab treatment. Mod Rheumatol 2011, 21(6):655-659.

12. Meintjes G, Lawn SD, Scano F, Maartens G, French MA, Worodria W, Elliott $J H$, Murdoch D, Wilkinson RJ, Seyler C, John L, van der Loeff MS, Reiss P, Lynen L, Janoff EN, Gilks C, Colebunders R, International Network for the study of HIV-associated IRIS: Tuberculosis-associated immune reconstitution inflammatory syndrome: case definitions for use in resource-limited settings. Lancet Infect Dis 2008, 8(8):516-523.

13. Shelburne SA, Montes M, Hamill RJ: Immune reconstitution inflammatory syndrome: more answers, more questions. J Antimicrob Chemother 2006, 57(2):167-170

14. Matsumoto T, Tanaka T, Kawase I: Infliximab for rheumatoid arthritis in a patient with tuberculosis. N Engl J Med 2006, 355(7):740-741.

doi:10.1186/1471-2334-14-114

Cite this article as: Takazono et al:: Paradoxical response to

disseminated non-tuberculosis mycobacteriosis treatment in a patient receiving tumor necrosis factor-a inhibitor: a case report. BMC Infectious Diseases 2014 14:114.

\section{Submit your next manuscript to BioMed Central and take full advantage of:}

- Convenient online submission

- Thorough peer review

- No space constraints or color figure charges

- Immediate publication on acceptance

- Inclusion in PubMed, CAS, Scopus and Google Scholar

- Research which is freely available for redistribution

Submit your manuscript at www.biomedcentral.com/submit
C) BioMed Central 